\title{
Spacecraft Navigation using the VLBA
}

\author{
Ed Fomalont* \\ National Radio Astronomy Observatory, Charlottesville, VA USA \\ E-mail: efomalonenrao.edu
}

\section{Tomas Martin-Mur, James Border, Charles Naudet, Gabor Lanyi \\ Jet Propulsion Laboratories, Pasadena, CA USA \\ E-mail: tomas.j.martinmur@jpl.nasa.gov}

\section{Jon Romney, Vivek Dhawan}

National Radio Astronomy Observatory, Socorro, NM USA

E-mail: jromney, vdhawanenrao.edu

\section{Barry Geldzahler}

National Aeronautics and Space Administration, Washington, DC USA

E-mail: bgeldzah@hq.nasa.gov

In 2008, the VLBA observed the Phoenix spacecraft for 8 sessions, each of about three hours, over a period of four months up to the Mars landing. Phase-referencing VLBI techniques were used between the Phoenix 8.4-GHz telemetry signal, the continuum emission from nearby quasars, and the telemetry signal from the two Mars orbiters. The astrometric results of these observations were compared with the accurate trajectory of Phoenix determined after landing in order to assess the VLBA positional accuracy and its feasibility for future NASA spacecraft tracking. The nominal celestial position of Phoenix was obtained to about 0.15 mas accuracy, but achieved an accuracy of 0.015 mas (15 meters at Mars) with respect to the Mars center of mass within two days of landing using in-beam observations of Phoenix and the two Mars orbiters.

10th European VLBI Network Symposium and EVN Users Meeting: VLBI and the new generation of radio arrays

September 20-24, 2010

Manchester UK

\footnotetext{
${ }^{*}$ Speaker.
} 


\section{Motivation for VLBI Tracking of Spacecraft}

Doppler and radar ranging methods obtain the accurate distance to a spacecraft, and these are the main navigational techniques used for the successful insertion of spacecraft on or around solarsystem bodies. However, the ability of VLBI techniques to determine the spacecraft position on the plane of the sky during its flight provides complementary positional information that is critical in some cases in determining the precise location of the spacecraft.

Supporting interferometric observations have been made for many years using the three DSN 70-meter antennas in Goldstone, CA, Robledo, Spain and Tidbinbilla, Australia. The observing technique measures the delay between quasars and spacecraft to determine their angular separation. However, to measure sufficiently accurate delays, the spacecraft must transmit special signals that are separated by many megaHertz [1]. The VLBA, with its accurate phase stability and observational flexibility, needs only to detect the spacecraft telemetry signal in order determine its relative position with respect to quasars nearby in the sky-in exactly the same manner as that used for natural radio sources that emit relatively narrow-band emission-using a technique called phase referencing $[2,3]$. Thus, spacecraft interferometry with the VLBA is less invasive to spacecraft operations since only the telemetry signal is needed. Angular separations between a spacecraft approaching a planet with other spacecraft orbiting or on the planet surface can also be obtained from their telemetry signals using VLBA phase referencing.

The astrometric goals of the 2008 VLBA observations were to determine the VLBI positional accuracy of Phoenix that could be achieved, and to begin the development of an operational system: obtaining accurate a priori parameters for the observations, determining the optimum observation scheme, correlating the interferometric data, processing the data to obtain images, converting the data into observed delays for input of the VLBA results into the navigational software package. Real-time connections from the antennas to the correlator were also investigated in order to obtain 12-hour turn-around from observations to results, when necessary for the mission.

\section{VLBA Observing Strategy and Reductions}

The observing scheme is illustrated in Fig. 1. The bulk of the observation time consisted of alternating one-minute scans: Quasar-Phoenix-Quasar-Orbiters-Quasar-Phoenix...etc. The quasars are compact ICRF sources with known position to 0.15 mas accuracy and within several degrees of Phoenix. During the three days before landing, Phoenix and the orbiters were closer than $6^{\prime}$ and could be observed simultaneously by the VLBA. The two Mars orbiters, Odyssey and Mars Reconnaissance Orbiter (MRO) were always sufficient close to be observed with one pointing.

The mean telemetry frequencies for Phoenix, MRO and Odyssey were 8.407, 8.439 and 8.550 $\mathrm{GHz}$, respectively, with a telemetry bandwidth of 50 to $300 \mathrm{kHz}$. Accurate spacecraft orbits were obtained from JPL navigation team and used in the correlator model to determine the residual visibility data. Corrections for solar and planetary gravitational bending and parallax and wave curvature were included. The data were averaged every $1 \mathrm{sec}$ and four $8 \mathrm{MHz}$ bandwidths, each with $256 \times 31.5 \mathrm{kHz}$ channels, covered the spacecraft signals. All spacecraft signals were strong ( 1 to $30 \mathrm{Jy}$ ) and point-like, whereas the quasars were about $0.2 \mathrm{Jy}$ so the entire $32 \mathrm{MHz}$ bandwidth was used to obtain good SNR. 


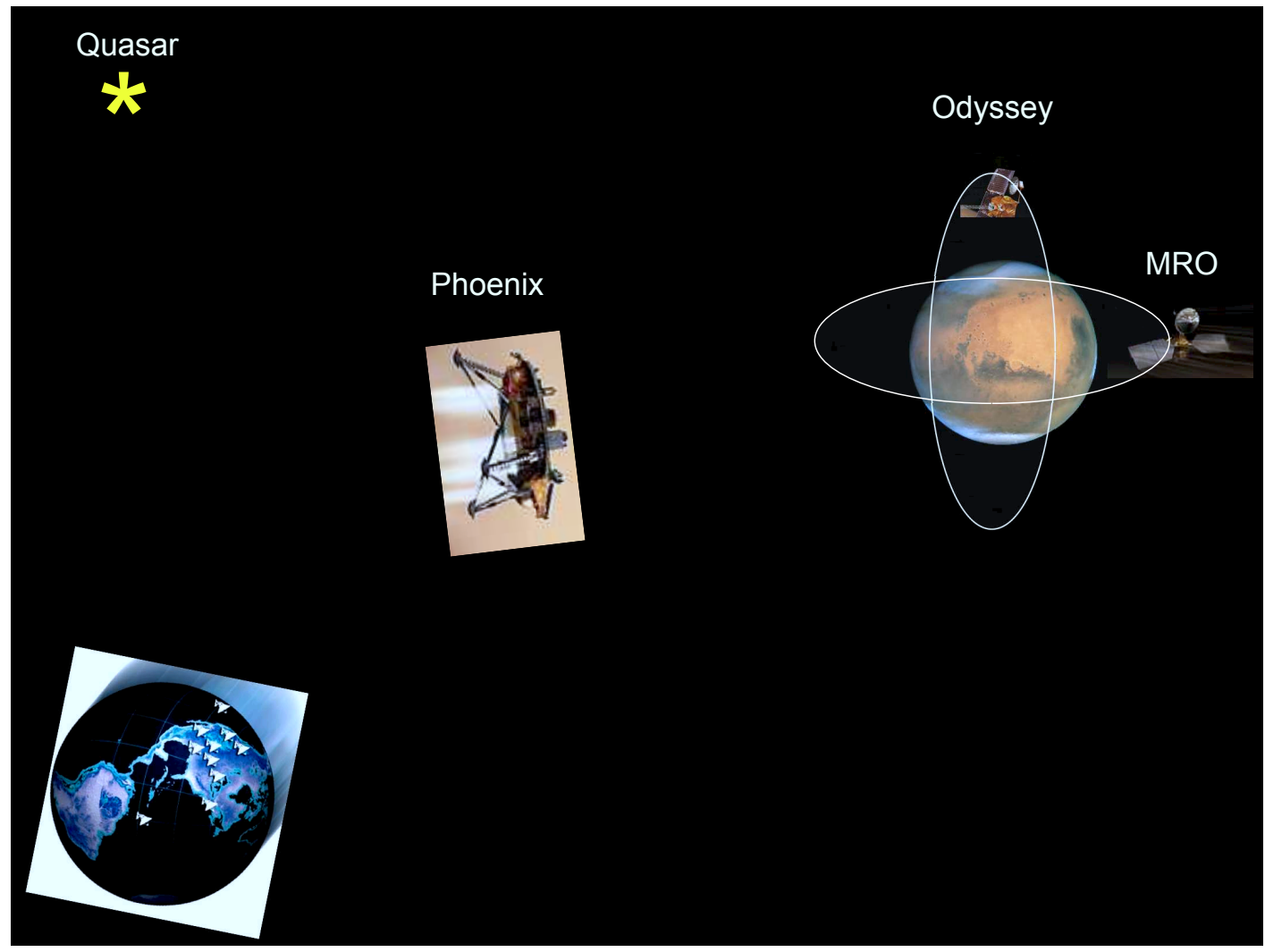

Figure 1: The Observing Scheme: The ten VLBA-antennas alternated observations of the quasar with Phoenix and the two Mars orbiters. Within two days of landing, Phoenix was sufficiently close to Mars so that all three spacecraft could be observed simultaneously within one antenna pointing area.

After data correlation initial a priori corrections, relevant data editing, and an ionosphere refraction model (taken from GPS observations) were applied to the visibility data. The quasar data were then used as the "primary reference source" in order to determine the instrumental antennabased delays and phases for each quasar scan. These values were interpolated in time to the spacecraft data to determine their calibrated visibility. The spacecraft data were then processed in two ways: First, using FFT and clean deconvolution methods, a spacecraft image was obtained with a resolution of about 1 mas. The offset of the peak intensity of the spacecraft image from the image center gives its residual position with respect to the assumed orbit used in the correlator model. The velocity estimates of the spacecraft orbits were sufficiently accurate, so that no blending of the image response occurred over each session of a few hours. Second, the visibility data were converted into a residual delay between the quasar and the spacecraft for each scan and for each antenna. When this delay is added to that assumed in the correlator model, the total delay between the quasar and spacecraft is obtained, and this quantity is free of any assumptions made in the correlator model and processing of the data. It is the time difference between the quasar and spacecraft wavefronts impinging on each antenna, compared with the wavefronts reaching the Earth geocen- 


\begin{tabular}{ccccc}
$\begin{array}{c}\text { Date } \\
2008\end{array}$ & $\begin{array}{c}\text { P-Q Sep } \\
(\mathrm{deg})\end{array}$ & $\begin{array}{c}\text { P-O Sep } \\
(\mathrm{deg})\end{array}$ & $\begin{array}{c}\text { P-Q Accuracy } \\
(\mathrm{mas})\end{array}$ & $\begin{array}{c}\text { P-O Accuracy } \\
(\mathrm{mas})\end{array}$ \\
\hline Mar 01 & 2.3 & 3.1 & $0.20+0.25$ & 0.33 \\
May 14 & 1.4 & 1.8 & $0.09+0.15$ & 0.20 \\
May 17 & 1.5 & 1.3 & $0.13+0.15$ & 0.14 \\
May 19 & 1.6 & 0.8 & $0.08+0.15$ & 0.07 \\
May 22 & 1.8 & 0.22 & $0.10+0.15$ & 0.05 \\
May 23 & 1.8 & 0.14 & $0.06+0.15$ & 0.03 \\
May 25 & 1.9 & 0.06 & $0.09+0.15$ & 0.02 \\
May 26 & 1.9 & 0.02 & $0.11+0.15$ & 0.015 \\
\hline
\end{tabular}

Table 1: Accuracy of the VLBA Phoenix Observations

ter. This delay is similar to spacecraft navigation ranging data and could easily be added to the navigation data base for processing and comparison of many types of navigation data, including the VLBA data [4].

\section{Navigation Analysis of VLBA Data}

Information concerning each of the eight observing sessions and the astrometric results are given in Table 1. The second column gives the Phoenix to Quasar separation (P-Q Sep) whereas the Phoenix-Orbiter separation (P-O Sep) decreased from 3.1 deg to less than one arcmin as the spacecraft approached the planet. The astrometric results given in the table have been derived from the JPL navigation software in which the VLBA-derived delay data were compared with the known Phoenix trajectory after landing. The Phoenix position accuracy with respect to the quasar (P-Q Accuracy) contains two terms. The first term is the relative position accuracy between the quasar and spacecraft, and depends on their angular separation in the sky and the quality (mostly weather related) of the data. The second term gives the estimated quasar position accuracy with respect to the ICRF celestial reference frame. The final column lists the position accuracy between Phoenix and the two orbiters (P-O Accuracy). This position difference is absolute in the sense of tying Phoenix to Mars since the relative position of the orbiters with respect to the center of mass of Mars is accurate to 0.001 mas. The spacecraft to spacecraft accuracy significantly improves as Phoenix approaches Mars and the day before landing, the estimated error of 0.015 mas corresponds to 15 meters at Mars.

\section{Conclusions}

The analysis of this extensive pilot program using the VLBA to track the Phoenix spacecraft demonstrates that the astrometric accuracy of spacecraft position with respect to the ICRF frame, using quasars, is about 0.2 mas. The entire ecliptic region can be adequately observed since the VLBA north-south positional accuracy degrades only slightly as far south as $-30^{\circ}$ declination. The use of the telemetry signals from the spacecraft provides the VLBA with the necessary flexibility to determine the spacecraft to spacecraft (or orbiter or landed spacecraft) separation using the same 
observational techniques as that for quasar phase referencing. When these spacecraft are located within a few arcminutes, their relative positions can determines as accurately as 0.01 mas.

During the 2008 observations, NRAO and JPL developed the infrastructure and communication needed for future collaboration: (1) The procurement of optical fiber links between most of the VLBA stations were investigated. This capability will be required to obtain astrometric data after critical maneuvers no more than 12 hours after the measurements. (2) In the near future, the main telemetry signals for deep space navigation will move to $33 \mathrm{GHz}$ for higher astrometric precision and less interference from plasma refraction. The possible upgrade of the VLBA antennas to dual $33 \mathrm{GHz} / 8 \mathrm{GHz}$ receiver capabilities to meet this frequency change was also investigated. (3) Efficient and semi-automatic methods were developed to transmit the spacecraft a priori orbits and other parameters from JPL to NRAO, to correlate and process the data quickly with robust scripts, to obtain images and total delay quasar-spacecraft differences, and to transport these data into the JPL navigational data base for further analysis.

With accurate tracking of spacecraft, the VLBA observations indirectly obtain precise positions of solar system objects. For example, recent VLBA tracking of the Cassini spacecraft has procured the most accurate position of the center of mass of Saturn [5]. Such observations can improve the ephemerides and dynamical understanding of the solar system objects.

\section{References}

[1] Lanyi, G., Bagri, D. S. \& Border, J. 2007, Angular Position Determination of Spacecraft by Radio Interferometry, Proc IEEE, 95, 2193-2201

[2] Napier, P. J., Bagri, D. S., Clark, B. G., Rogers A. E. E., Romney, J. D., Thompson, A. R. \& Walker, R. C. 1994, The Very Long Baseline Array, Proc. IEEE, 82, 658-672

[3] Pradel, N., Charlot, P. \& Lestrade, J.-F. 2006, Astrometric Accuracy of Phase-referenced Observations with the VLBA and EVN, A\&A, 452, 1099-1106

[4] Martin-Mur, T. et al, 2006 Use of Very Long Baseline Array Interferometric Data for Spacecraft Navigation, Proceedings of the $25^{\text {th }}$ International Symposium on Space Technology and Science, Kanazawa, Japan, May 4-11, 2006

[5] Jones, D L., Fomalont, E., Dhawan, V., Romney, J., Folkner, W. M., Lanyi, G., Border, J. \& Jacobson, R. 2011, Astrometric Observations of Cassini with the VLBA, AJ, 141, in press 